\title{
HOME AUTOMATION SYSTEM USING ESP8266 MICROCONTROLLER AND BLYNK APPLICATION
}

\author{
I. Visan ${ }^{1}$, E.M. Diaconu ${ }^{2}$ \\ Valahia University of Targoviste, Faculty of Electrical Engineering, Electronics and Information Technology \\ E-mail: emy_diaconu@yahoo.com
}

\begin{abstract}
This paper proposes the creation of a home automation system using an ESP8266 microcontroller and the Blynk application. The system consists of 2 sensors responsible for measuring the temperature/humidity and the level of brightness in the building, a 4-channel relay module responsible for controlling 4 devices/appliances/electrical installations and an LED that demonstrates its control through a PWM signal provided by the ESP8266 microcontroller. Using the Blynk application this system can be controlled and monitored via a Wi-Fi connection directly on the mobile device which makes this system an IoT (Internet of Things) one. The programming of the system was done in the Arduino IDE program using the already existing libraries for the sensors and for the Blynk application. The mobile application used for controlling and monitoring the system is able to display information about temperature /humidity, brightness and on the control side, the application allows the user to individually operate each channel of the relay module and the LED.
\end{abstract}

Keywords: IoT, ESP8266, Blynk

\section{INTRODUCTION}

The development of the internet has changed society and the biggest change is happening at this moment. More and more technologies are converging to create a real revolution when it comes to the Internet by using more and more objects that have their own connection and identity in the web space. The Internet of Things (IoT) is the next phase of development that takes place after the Internet of computers and this phase of development contributes to the connection more or less of all objects that are connected and managed in the virtual world. [1] The greatest expansion of the Internet is the IoT universe and its radical effects can be seen in every industry and in our daily lives. In the near future more and more devices and systems will be able to send and receive information via the Internet and this involves more and more new developments that have enormous potential, for example, in business markets. IoT will allow users to connect to the market and to sectors that in the past did not have business relationships, generating new products and services that lead directly to the creation of new business models. In order to create a partner base, existing web platforms can be used, which would help to expand or complete their offer in various new ways. The IoT concept is not a distant vision of the future but is already used in many fields and has a great impact on the development of technology. For every device that has the ability to connect to the Internet, an IP address is needed, and it is predicted that by 2022 there will be up to 100 billion devices connected to the Internet for a population of 7.6 billion people. According to an existing study on urban environments, it results that each human being is surrounded by up to 5000 connected devices and this leads to the transition to IPv6 to ensure the very large number of possible addresses for these devices [2]. The principle of home automation involves monitoring and controlling devices through a unified system. The control systems consist of temperature control, lighting control, burglar alarm system and appliances. The principle of home automation is related to the automation of industrial buildings, for example, large shopping centers. [3] Different types of systems are available for home automation, being designed and purchased for different purposes. One of the biggest problems for home automation is the lack of interconnection and interoperability between different devices. As a general example, inoperable and interconnected systems range from switches used for lighting controlled by a remote WiFi or Bluetooth connection to complete internet-connected systems that have the role of controlling all equipment in an entire building.[4] Home automation is an extremely attractive field of IoT. These areas represent how the sensors and actuators in a building are configured, connect automatically and provide remote control by summing up a multitude of applications for control and monitoring through different connection modes. Such connection modes are offered by IoT device manufacturers but the internet connection function is sometimes missing, thus requiring a special auxiliary hardware device connected to an internet connection via the IPv4 protocol that offers the possibility of internet-device connections. By connecting the devices directly to the internet or through the special auxiliary hardware it allows the control of these devices remotely using PCs, mobile devices through a suitable software application[5].

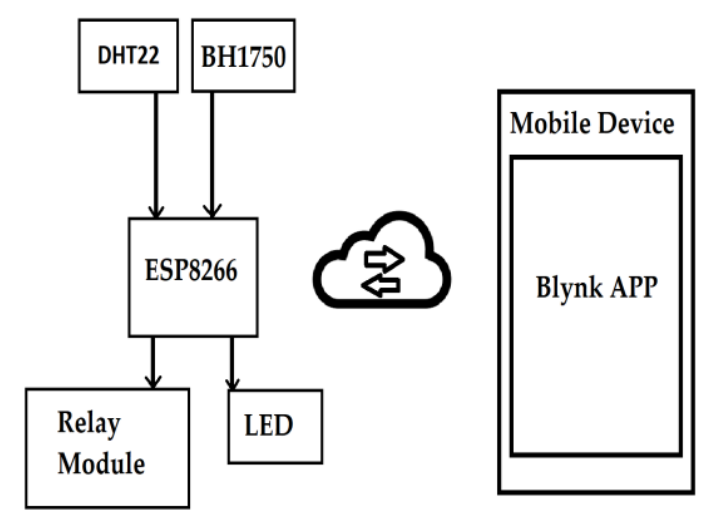

Figure 1. Home automation system diagram 
The system diagram is showed in Figure 1 and it consists of 2 sensors responsible for measuring the temperature /humidity (DHT22) and the level of brightness (BH1750) in the building, a 4-channel relay module is responsible for controlling 4 devices/appliances/electrical installations and an LED that demonstrates its control through a PWM signal provided by the ESP8266 microcontroller. Using the Blynk application this system can be controlled and monitored via a Wi-Fi connection directly on the mobile device. The mobile application used for controlling and monitoring the system is able to display information about temperature/humidity, brightness and on the control side, the application allows the user to individually operate each channel of the relay module and the LED.

\section{HARDWARE DESCRIPTION}

\subsection{ESP8266 microcontroller}

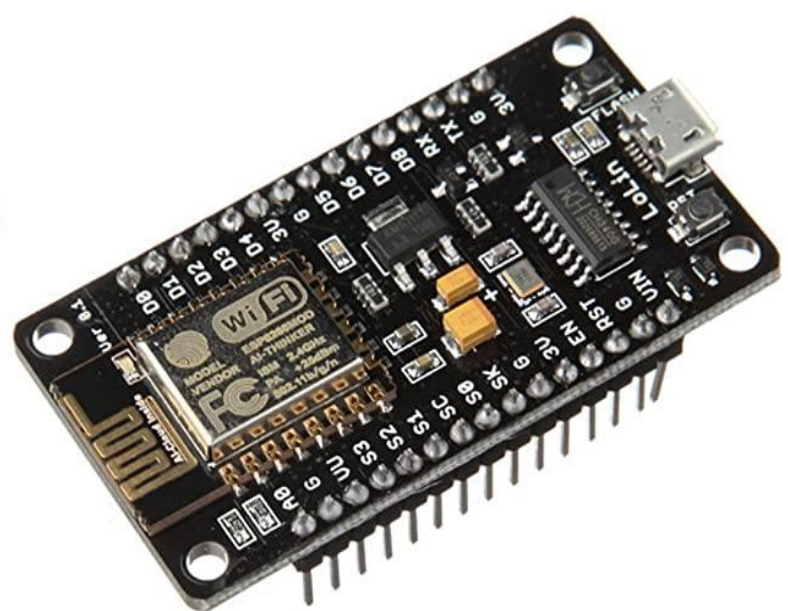

Figure 2. ESP8266 microcontroller.

The microcontroller (Figure 2) comes equipped with the ESP-12E module, this module contains the ESP8266 chip which has a 32-bit microprocessor with a RISC architecture. The found microprocessor is a Tensilica Xtensa that supports RTOS and has an operating frequency between $80 \mathrm{MHz}$ and $160 \mathrm{MHz}$. To store the data and programs developed for this microcontroller, the internal FLASH memory is used, which can accommodate a quantity of $4 \mathrm{MB}$. On the connection side, the microcontroller comes equipped with Wi-Fi and Bluetooth and supports the Deep Sleep function which makes the microcontroller a logical choice for our IoT project.

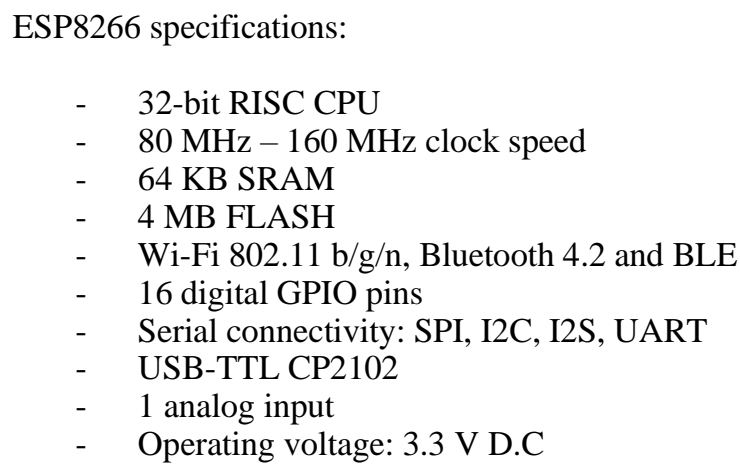

- Input voltage: maximum 12 V D.C

\subsection{DHT22 temperature / humidity sensor}

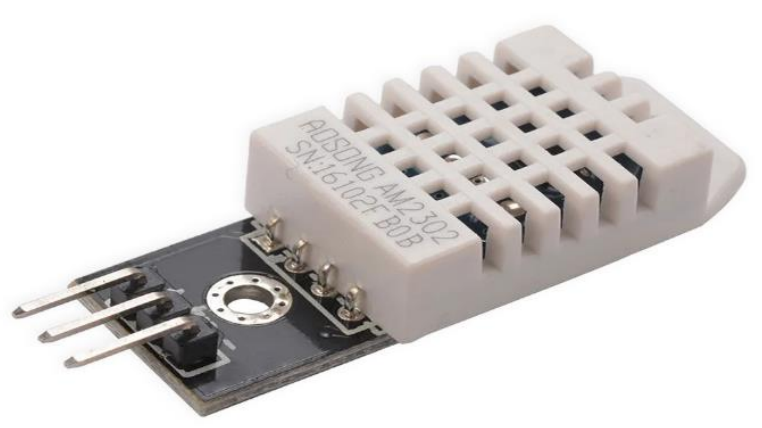

Figure 3. DHT22 temperature/humidity sensor.

The DH22 digital sensor (Figure 3) is designed to measure basic temperature and humidity at a low cost. To measure humidity, it uses a capacitive humidity sensor and to measure temperature it uses a thermistor to measure the air around it. The sensor provides a digital signal through the output pin and its use is intuitive but requires good synchronization to obtain data being one of the real disadvantages. The new data is obtained at a period of 2 seconds, which makes the measurements obtained 2 seconds old.

DHT22 specifications:

- Current consumption: $2.5 \mathrm{~mA} \max$

- Operating voltage: $3 \mathrm{~V}-5 \mathrm{~V}$ D.C

- Humidity readings: $0 \%-100 \% \quad(2 \%-5 \%$ accuracy)

- Temperature readings: $-40^{\circ} \mathrm{C}-80{ }^{\circ} \mathrm{C}\left( \pm 0.5^{\circ} \mathrm{C}\right.$ accuracy)

- $\quad$ Sampling rate: $0.5 \mathrm{~Hz}$

\subsection{BH1750 ambient light sensor}

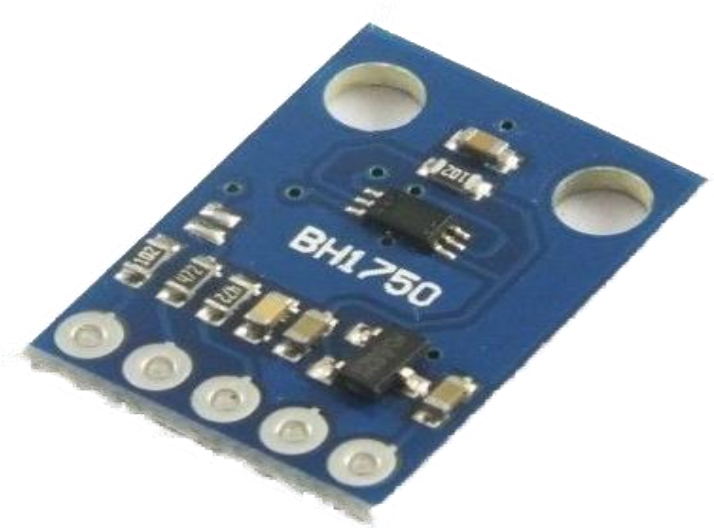

Figure 4. BH1750 ambient light sensor. 
The BH1750 sensor (Figure 4) is used to measure light intensity and it is frequently used to adjust the brightness of the display for mobile devices, LCD screens or as a light sensor in the automotive industry. To communicate with the ESP8266, this sensor uses the I2C communication protocol via SCL and SDA pins. The LUX value offered by this sensor is performed directly without the need for calculation formulas in the source code. The LUX value is measured according to the amount of light that the sensor captures, without depending on the light source or being influenced by IR radiation.

\section{BH1750 specifications:}

- $\quad$ Operating voltage: $2.4 \mathrm{~V}-3.6 \mathrm{~V}$

- Current consumption: $0.12 \mathrm{~mA}$

- Accuracy: $\pm 20 \%$

- Communication protocol: I2C

- Measuring range: 1 LUX - 65535 LUX

\subsection{4-channel relay module}

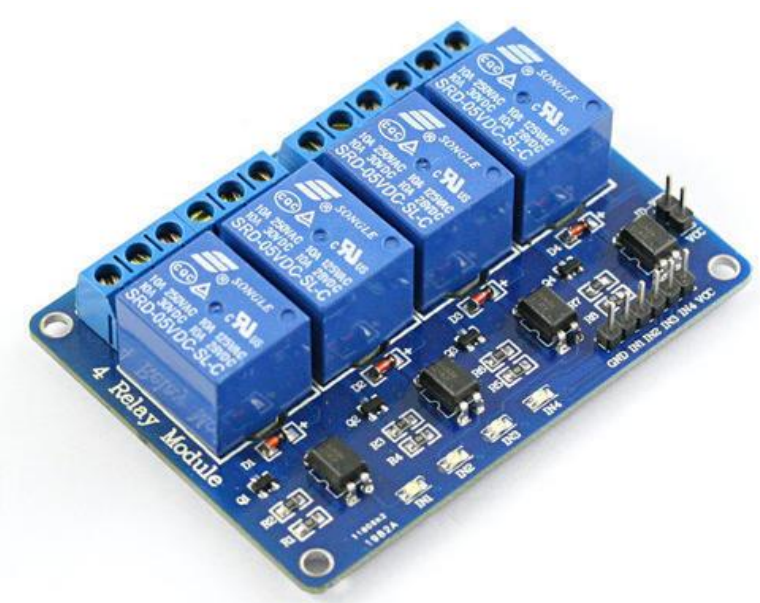

Figure 5. 4-channel relay module.

The 4-channel relay module (Figure 5) is a practically a switch that is electrically operated by supplying a coil with a small amount of voltage provided by the microcontroller, thus providing a magnetic field that attracts or rejects an electrical contact. The module is used to start or stop a circuit that uses a voltage or current much higher than the one supported by ESP8266. There is no direct contact between the low voltage electronic circuit and the high voltage electrical circuit because there are protected from each other. Each of the 4 channels has 3 types of connections called NC (Normal Closed), NO (Normal Open) and COM (Common). The relay module is used high current switching, isolated power delivery and home automation.

Relay module specifications:

- Operating voltage: $3.75 \mathrm{~V}-6 \mathrm{~V}$

- Quiescent current consumption: $2 \mathrm{~mA}$

- Current consumption when relay is active: $\sim 70 \mathrm{~mA}$

- Maximum contact voltage: 250V A.C, 30V D.C
- Maximum contact current: 10A

- $\quad$ Number of channels: 4

\section{BLYNK APPLICATION}

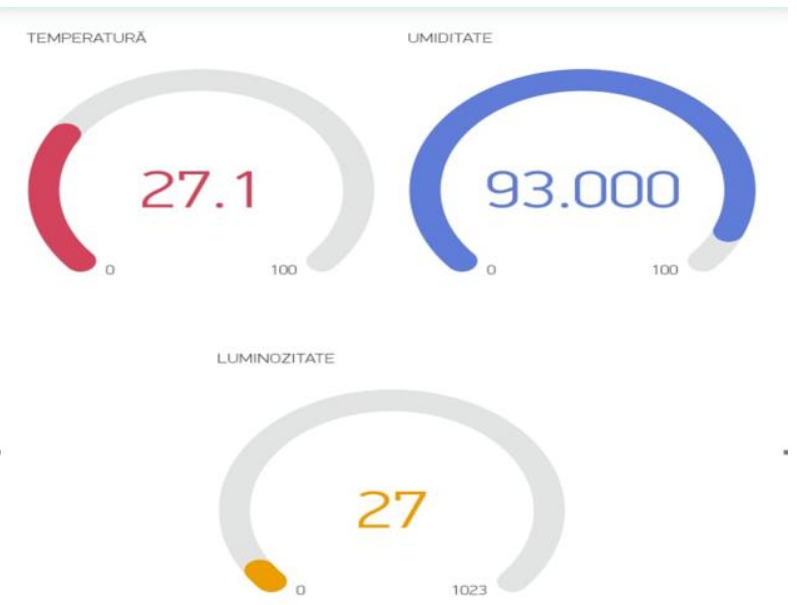

Figure 6. Temperature, humidity and brightness widgets.

The top part of the Blynk application (Figure 6) is made from 3 widgets. The red widget is used for showing the temperature measured by the system, the blue one is used for showing the humidity measured by the system and the yellow one is used for showing the ambient light level.

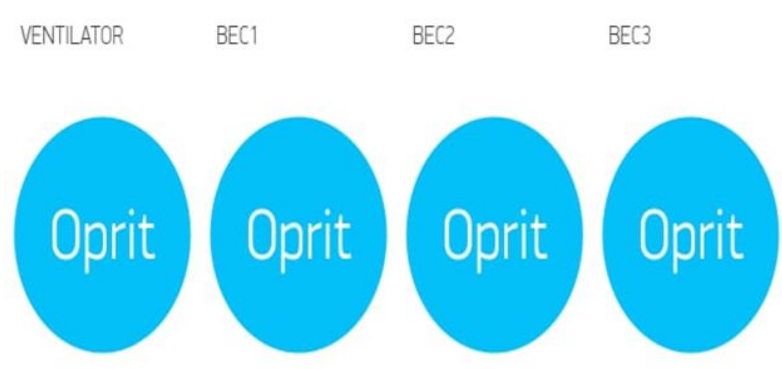

PWMLED

III

Figure 7. Relay channel buttons and LED PWM slider.

The bottom part of the Blynk application (Figure 7) is made from 4 buttons and a slider. Each button is used to operate one of the 4 channels on the relay module and the slider is used to provide a PWM signal that has the value from 0 to 255 to the LED, thus turning on the LED and setting up the brightness level of it.

\section{SYSTEM PROTOTYPE}

For the design of the prototype, we used Tinker CAD which is used for $3 \mathrm{D}$ modeling. The $3 \mathrm{D}$ model was made to represent a compact proof of concept module. The 3D 
model of the module was printed using a 3D printer and red PLA printing material, the print is made using a 0.05 $\mathrm{mm}$ layer height for best quality. The printed module accommodates the electronic connection board made from the ESP8266 microcontroller and connector for the sensors, relay module and LED.

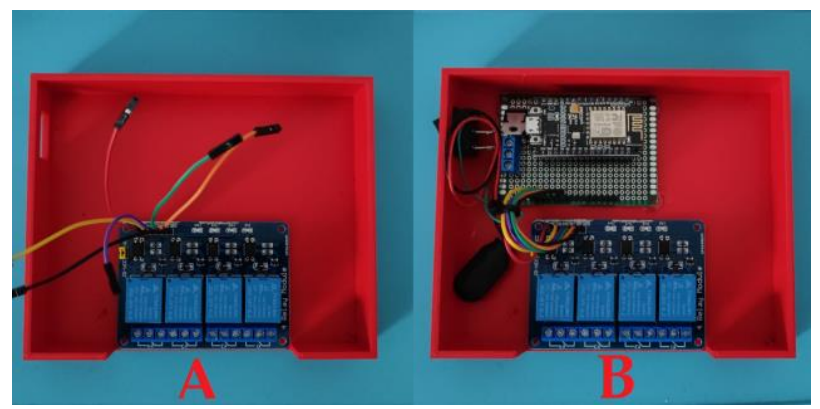

Figure 8. Relay module (A) and electronic connection board (B).

The electronic connection board and relay module are shown in Figure 8. The relay is showed on the left of the figure (A) and the connection board with the relay module connected to the board are showed on the right of the figure (B).

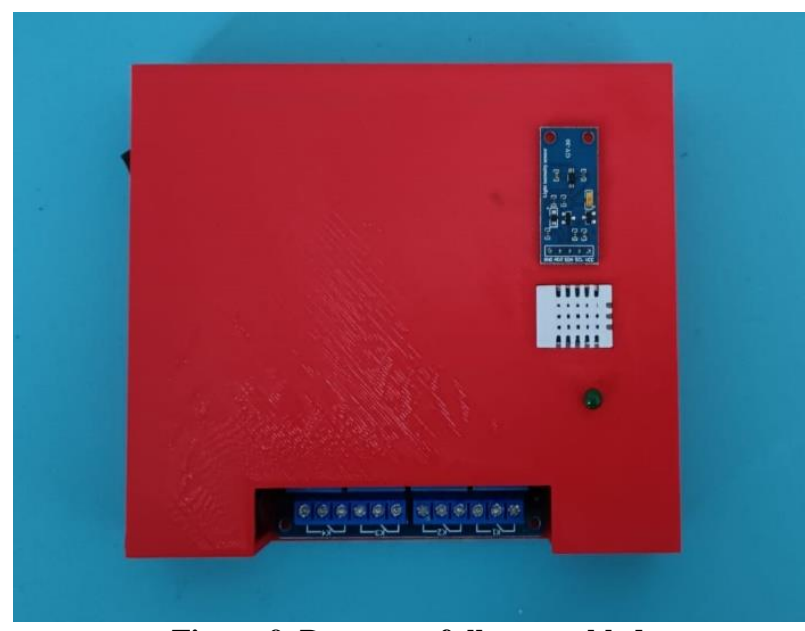

Figure 9. Prototype fully assembled.

The two sensors and the LED are mounted on the front of the case, also the front of the case has an opening for the relay module connectors providing easy access for connecting electrical equipment. To be mentioned this prototype of the system was made for presentation purpose only.

\section{CONCLUSIONS}

The biggest obstacle often encountered in the implementation of commercial smart home systems is the very high cost for such a system. Existing systems on the market often require additional network adaptors such as hubs which in turn raise the total cost of the system. This paper has studied and revised the currently available home automation system, using the ESP8266 development platform and the Blynk IoT platform so that an economical system can be achieved. Above all, this system offers excellent convenience to the user, as it makes it possible to control the devices connected to the relay module and monitor the temperature, humidity and brightness from a remote location via a $\mathrm{Wi}-\mathrm{Fi}$ internet connection using a mobile type that is available on Android and iOS operating systems.

\section{REFERENCES}

[1] The Smarthome Book: Simple ideas to assist with your smarthome renovation, Andrew Howe, Independently published, 15 Oct. 2018; ISBN 10: 1728785154, ISBN 13: 978-1728785158.

[2] Smart Homes in easy steps: master smart technology for your home, Nick Vandome, In Easy Steps Limited; Illustrated edition, 28 Aug. 2018; ISBN-10: 1840788259, ISBN-13: 978-1840788259.

[3] E. Diaconu, "Controlul sistemelor electronice", Targoviste, Romania, Valahia University Press, ISBN 978-606-603-170-7.

[4] Home Automation with Arduino: Automate your Home using Open-Source Hardware, Marco Schwartz, CreateSpace Independent Publishing Platform; 2nd edition, 30 July 2013, April 2021, ISBN-10: 1491016825, ISBN-13: 978-1491016824.

[5] V Measurement \& Control using Smartphone \& Tablet, Hans J. Berndt, Independently published, 4 Oct. 2017, ISBN-10: 1549604325, ISBN-13: 9781549604324 